\title{
Monte Carlo approach to the simulation of ethylene-propylene oxide polymerization and copolymerization.
}

Riccardo Tesser ${ }^{1, *}$, Elio Santacesaria ${ }^{2, *}$

${ }^{1}$ NICL - Department of Chemical Sciences, University of Naples Federico II, Via Cinthia 21, 80126, Naples, Italy

${ }^{2}$ CEO of Eurochem Engineering LtD, Via Codogno 5, 20139, Milano Italy;

www.eurochemengineering.com

*Corresponding authors E-Mails: riccardo.tesser@unina.it;

elio.santacesaria@eurochemengineering.com

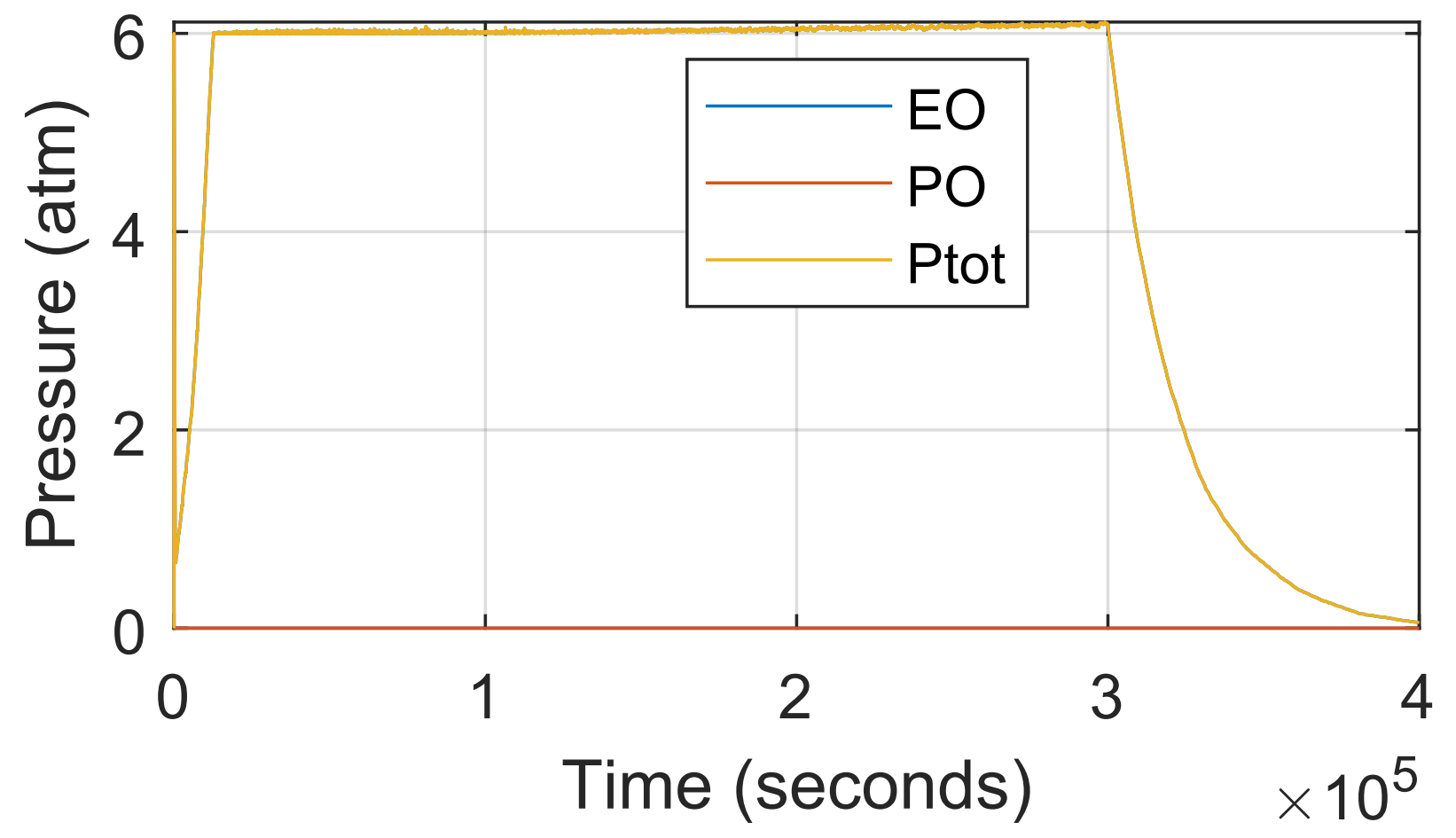

Figure S1 - Trend of the pressure during the reaction obtained by simulation of the system EG-EOhomopolymerization. 


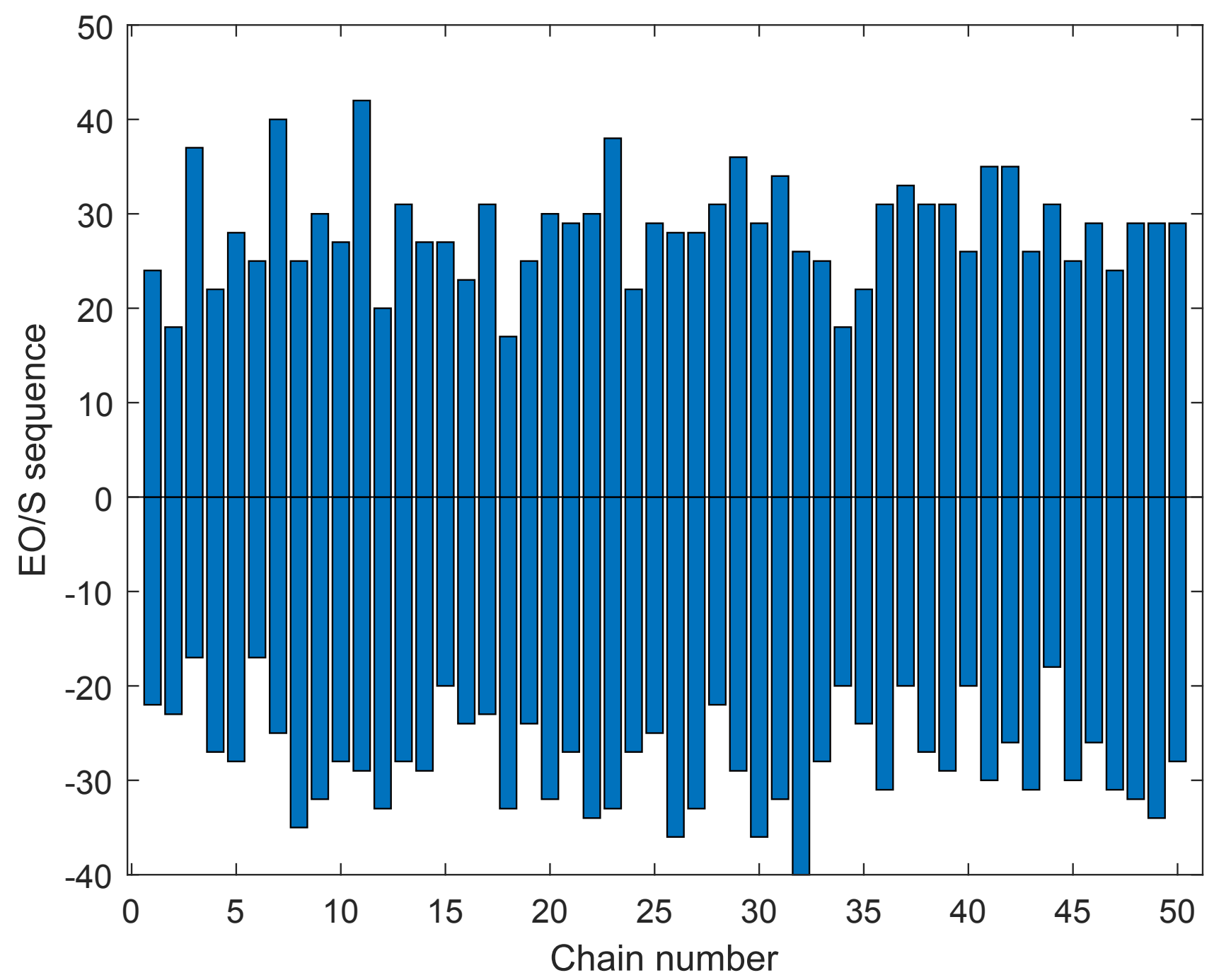

Figure S2 - Structural conformation of the obtained EO homopolymer. The zero line represent the substrate and, for a subset of 50 polymer chains, are represented the length of EO sequences as positive or negative bars on the two hydroxyls of the EG substrate. 

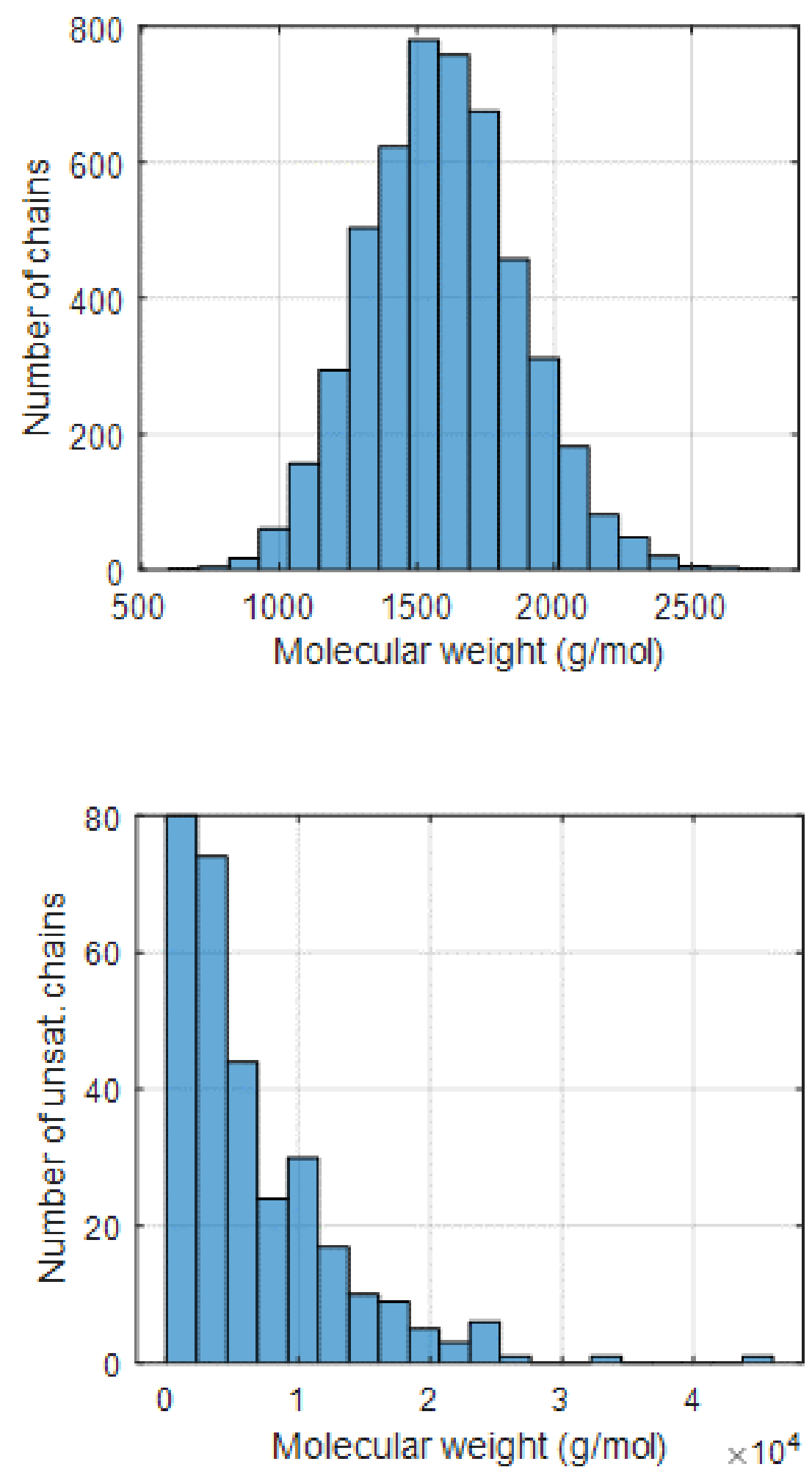

Figure S3 - Molecular weights distributions of respectively standard and unsaturated chains for an EG-EO-PO random copolymer. 